Supporting Information

\title{
Laser-Responsive Polymeric Nanomicelles to Subdue Tumor \\ Multidrug Resistance Based on Mild Photodynamic Therapy and \\ Chemotherapy
}

\begin{abstract}
Huangyong Jiang ${ }^{1}$, Yue $\mathrm{Su}^{1}, \mathrm{Nan} \mathrm{Li}^{2,3,4, *}$, and Xin $\mathrm{Jin}^{1, *}$
1 State Key Laboratory of Metal Matrix Composites, School of Chemistry and Chemical Engineering, Shanghai Jiao Tong University, Shanghai 200240, P. R. China. E-mail: jxcindy@sjtu.edu.cn

2 Department of Nuclear Medicine, Fudan University Shanghai Cancer Center, Shanghai, China. E-mail: linan466@163.com

${ }^{3}$ Shanghai Key Laboratory of Molecular Imaging, Shanghai University of Medicine and Health Sciences, Shanghai 201318, China.

${ }^{4}$ Department of Oncology, Shanghai Medical College, Fudan University, Shanghai, China
\end{abstract}




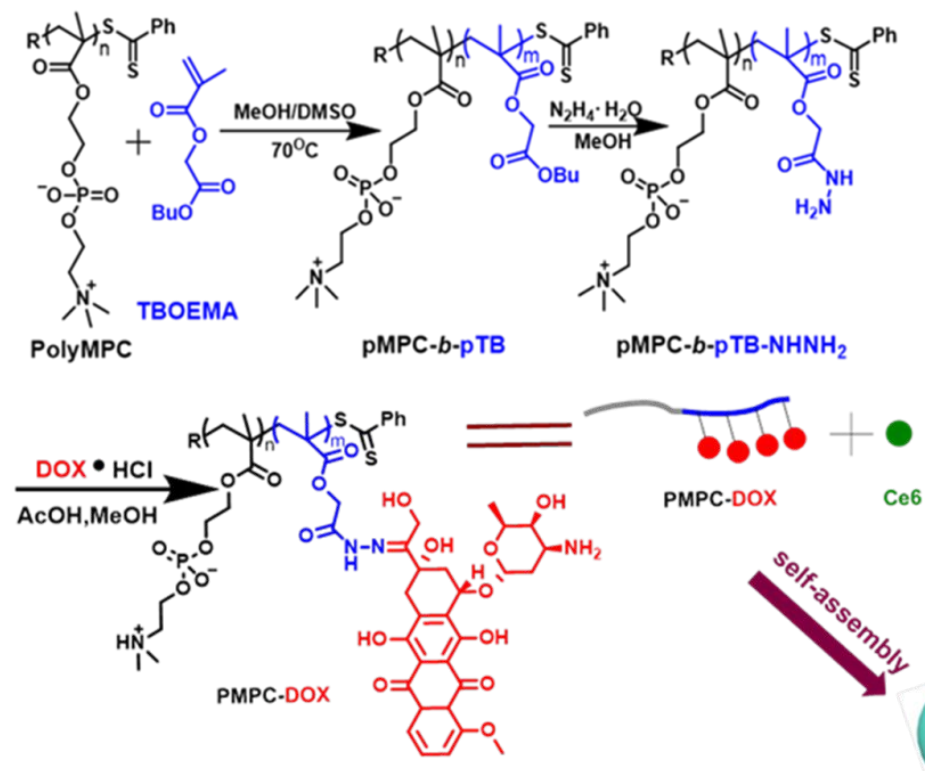

Ce6/pMPC-DOX micelles

Supporting information Figure S1: The formation of Ce6/pMPC-DOX micelles. 

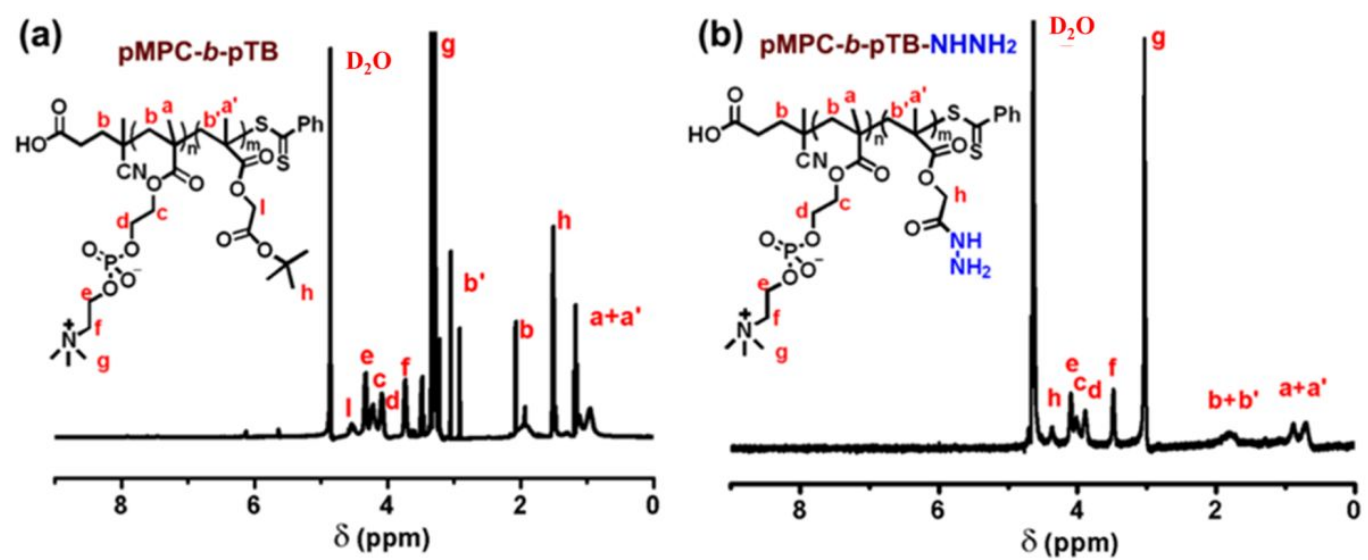

Supporting information Figure S2: ${ }^{1} \mathrm{H}$ NMR spectrum of pMPC-b-pTB (A) and pMPC-b-pTB-NHNH $\mathrm{H}_{2}$ (B) in $\mathrm{D}_{2} \mathrm{O}$.

As revealed by NMR in Fig.S2, the characteristic peaks at $4.56 \mathrm{ppm}$ were assigned to the methylene of $-\mathrm{O}-\mathrm{CH} 2-\mathrm{CO}$ - in $\mathrm{TB}$, suggesting the successful synthesis of monomer TB. And the new peaks at $1.34 \mathrm{ppm}$ could be assigned to TB units, confirming the successful synthesis of pMPC- $b$-pTB.

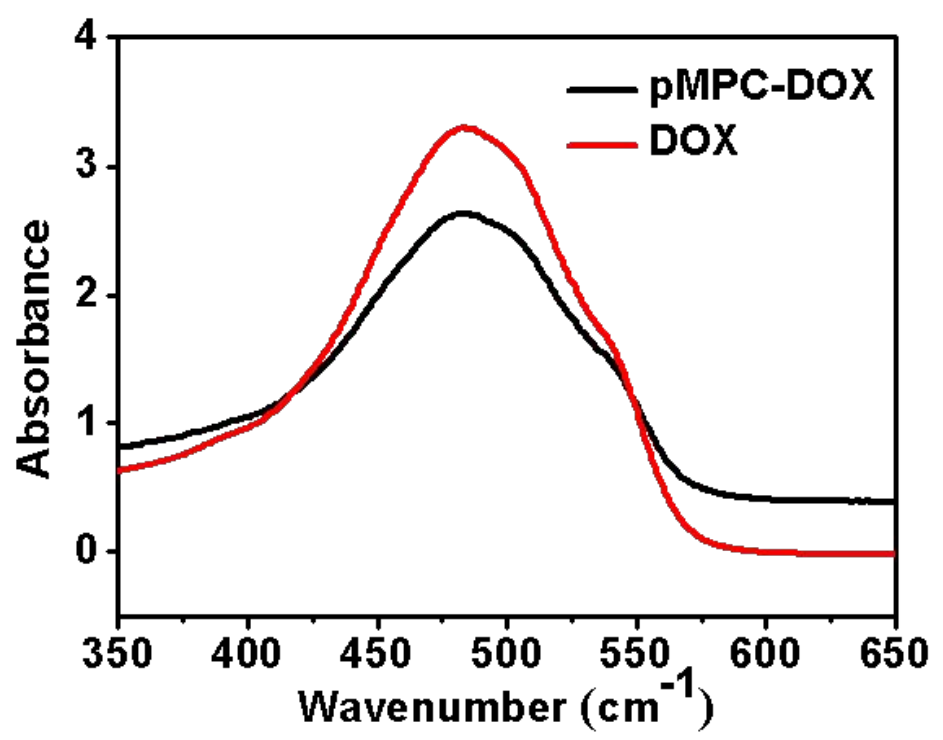

Supporting information Figure S3: UV-vis spectrum of pMPC-DOX and DOX hydrochloride in water.

As shown in Fig. S3, the UV-vis absorption spectrum of purified pMPC-DOX 
display characteristic absorption peaks of DOX at $480 \mathrm{~nm}$, indicating that DOX linked to the diblock polymers successfully.
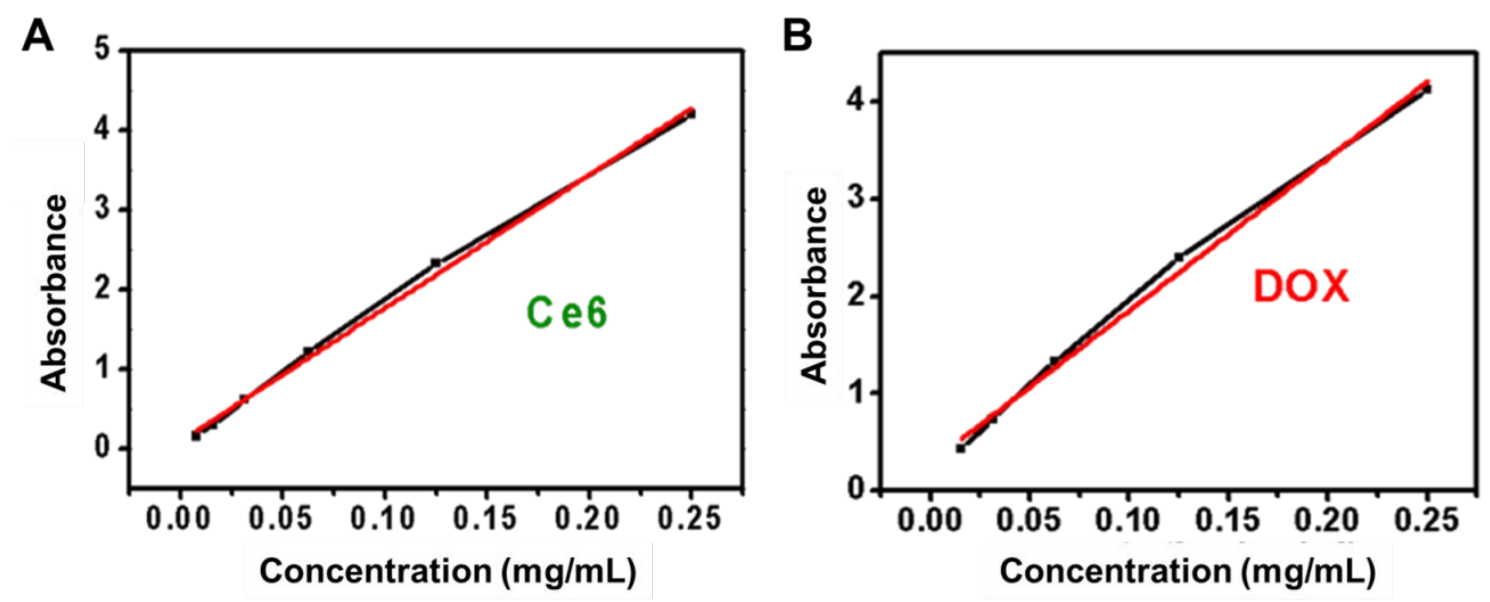

Supporting information Figure S4: (A) The standard curve of Ce6 hydrochloride at $660 \mathrm{~nm}$; (B)

The standard curve of DOX hydrochloride at $410 \mathrm{~nm}$.

As displayed in Fig. S4, the standard curve was conducted by the relationship between the absorbance value at $480 \mathrm{~nm} / 650 \mathrm{~nm}$ and the concentration of DOX/Ce6. The specific DOX/Ce6 loading calculating from the standard curve were shown in Table S1.

Supporting information Table S1. Characterization of Ce6/pMPC-DOX.

\begin{tabular}{|c|c|c|c|c|c|}
\hline samples & $\begin{array}{c}\text { DOX } \\
(w t \%)^{\mathrm{a}}\end{array}$ & $\begin{array}{c}\mathrm{Ce} 6 \\
(\mathrm{wt} \%)^{\mathrm{a}}\end{array}$ & $\begin{array}{c}\text { Size } \\
(\mathrm{DLS}, \mathrm{nm})^{\mathrm{b}}\end{array}$ & $\begin{array}{c}\text { Size } \\
(\mathrm{TEM}, \mathrm{nm})^{\mathrm{c}}\end{array}$ & $\begin{array}{l}\text { Zeta } \\
(\mathrm{mV})^{\mathrm{d}}\end{array}$ \\
\hline pMPC-DOX & 14 & l & $\sim 225$ & $\sim 191$ & 0.03 \\
\hline Ce6/pMPC-DOX & 14 & 7.6 & $\sim 120$ & $\sim 91$ & -4.6 \\
\hline
\end{tabular}




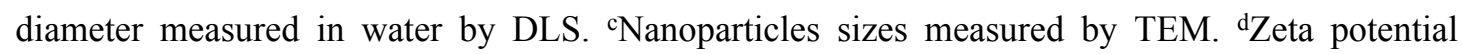
measured in water by Zetasizer.
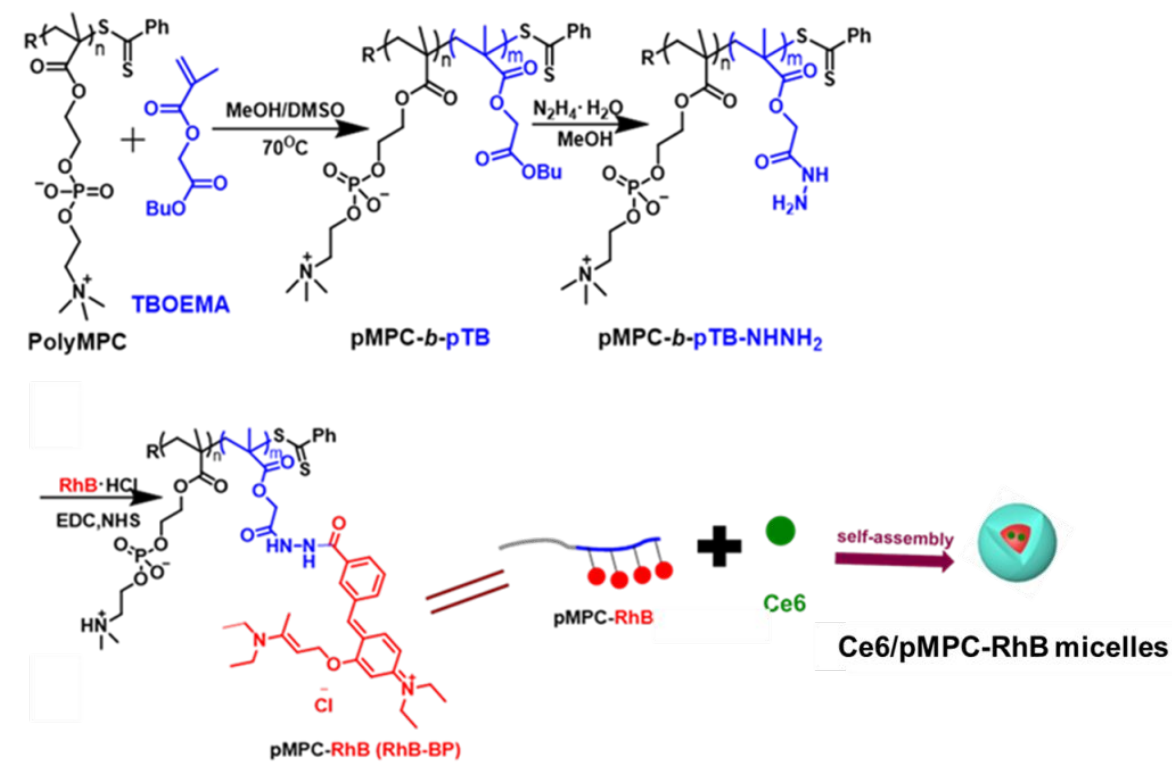

Supporting information Figure S5: The formation of Ce6/pMPC-RhB micelles.

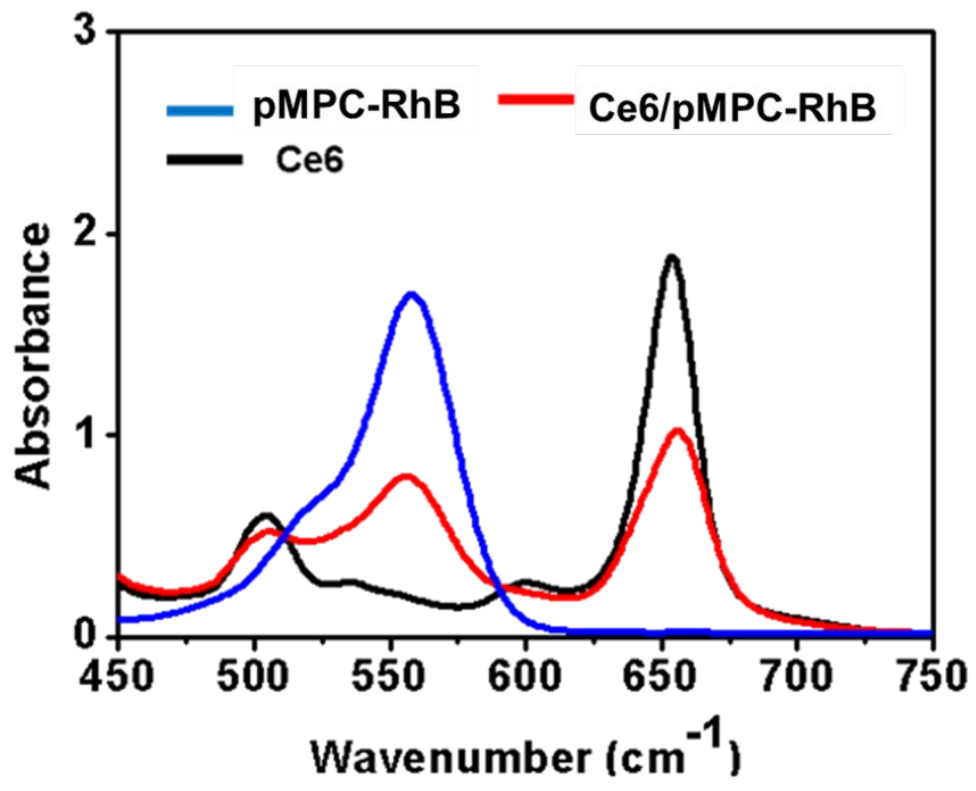

Supporting information Figure S6: UV-vis spectra of Ce6/pMPC-RhB micelles, free RhB and free $\mathrm{Ce} 6$ in mixed solution (DMSO: $\mathrm{H}_{2} \mathrm{O}=1: 1$ ).

As shown in Fig. S6, the UV-vis absorption spectrum of Ce6/pMPC-RhB samples display characteristic absorption peaks of $\mathrm{RhB} / \mathrm{Ce} 6$ at $550 / 650 \mathrm{~nm}$, indicating that 
$\mathrm{RhB}$ and Ce 6 was integrated into the diblock polymers successfully.

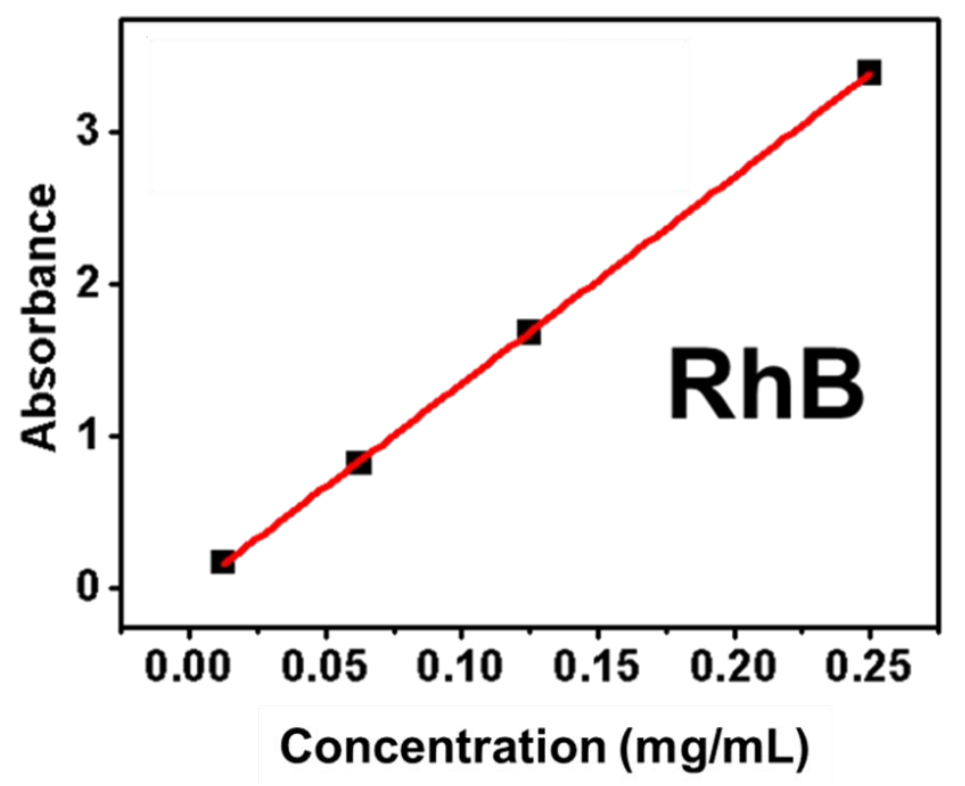

Supporting information Figure S7: The standard curve of RhB at $560 \mathrm{~nm}$.

As displayed in Fig. S7, the standard curve was conducted by the relationship between the absorbance value at $560 \mathrm{~nm}$ and the concentration of $\mathrm{RhB}$. The specific RhB loading calculating from the standard curve were shown in Table S2.

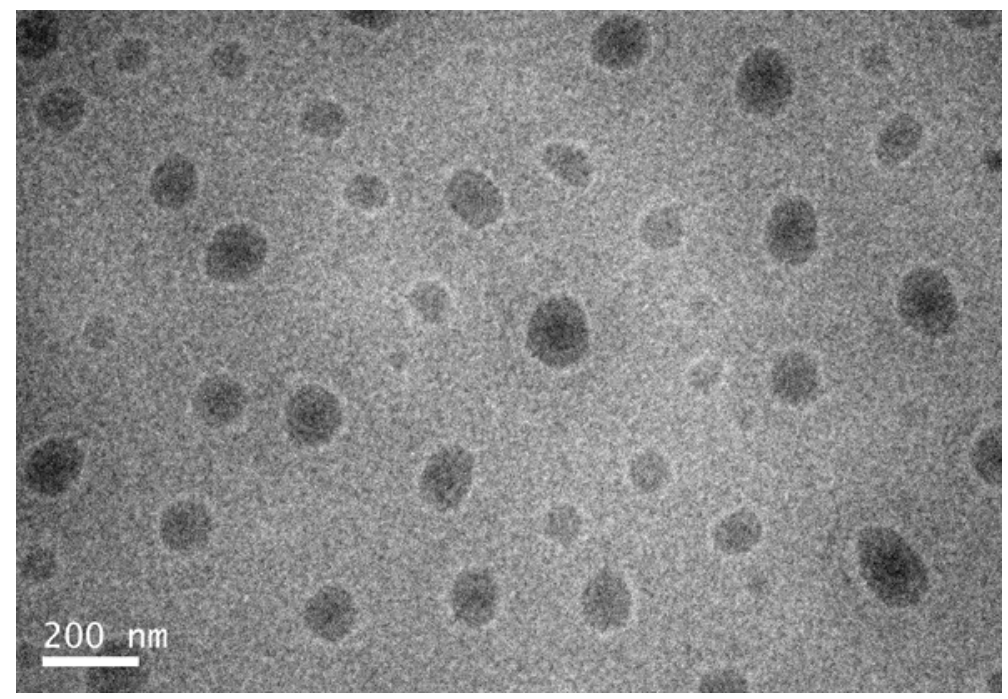

Supporting information Figure S8: TEM image of Ce6/pMPC-RhB. 
Supporting information Table S2. Characterization of Ce6/pMPC-RhB.

$\begin{array}{cccccc}\text { samples } & \text { RhB } & \text { Ce6 } & \text { Size } & \text { Size } & \text { Zeta } \\ & (\mathrm{wt} \%)^{\mathrm{a}} & (\mathrm{wt} \%)^{\mathrm{a}} & (\mathrm{DLS}, \mathrm{nm})^{\mathrm{b}} & (\mathrm{TEM}, \mathrm{nm})^{\mathrm{c}} & (\mathrm{mV})^{\mathrm{d}} \\ \text { pMPC-RhB } & 19 & / & \sim 190 & \sim 165 & -0.01 \\ \text { Ce6/pMPC-RhB } & 19 & 8.3 & \sim 169 & \sim 138 & -2.1\end{array}$

${ }^{\mathrm{a}} \mathrm{RhB}$ (Ce6) loading is obtained by UV-vis spectroscopy at 560 (660) $\mathrm{nm} .{ }^{b}$ Hydrodynamic

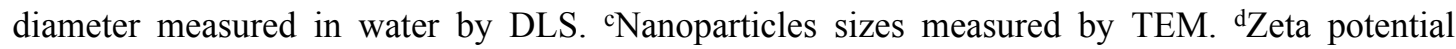
measured in water by Zetasizer.

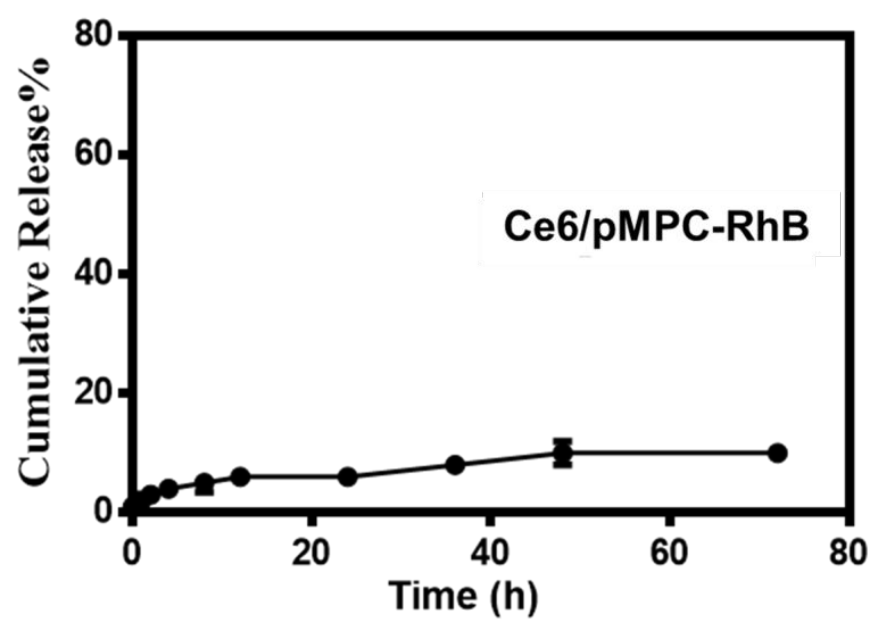

Supporting information Figure S9: RhB release profiles from Ce6/pMPC-RhB in acidic PBS solution $(\mathrm{pH}=5.0)$.

As displayed in Fig. S9, since Ce6/pMPC-RhB nanomicelles shown high stability in acidic condition $(\mathrm{pH}=5.0), \mathrm{Ce} / \mathrm{pMPC}-\mathrm{RhB}$ nanomicelles will not release $\mathrm{RhB}$ in cells and the fluorescence intensity of $\mathrm{RhB}$ in cells can represent the intracellular location of Ce6/pMPC-RhB nanomicelles. 


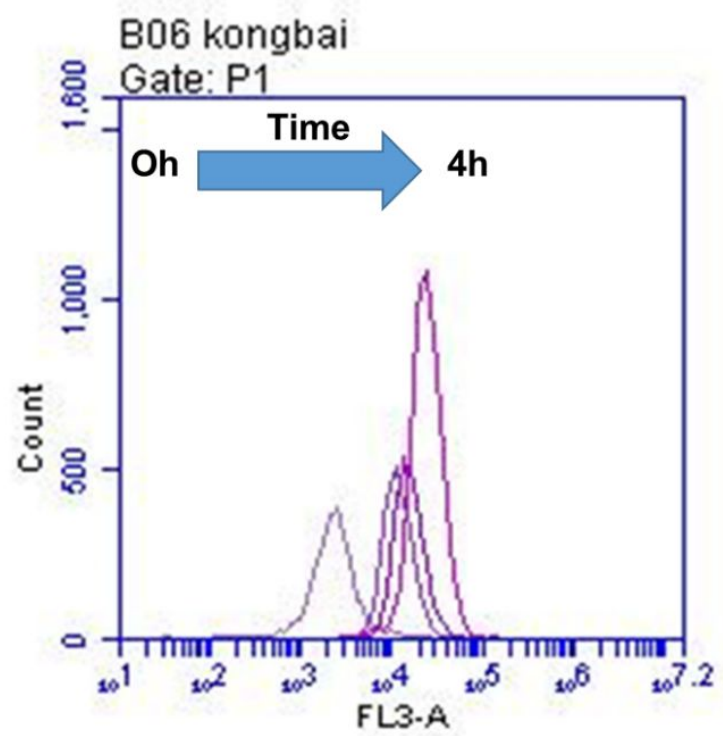

Supporting information Figure S10: FCM histogram profiles of MCF-7/ADR cells incubated with Ce6/pMPC-RhB micelles for 0h, 1h, 2h and 4h.

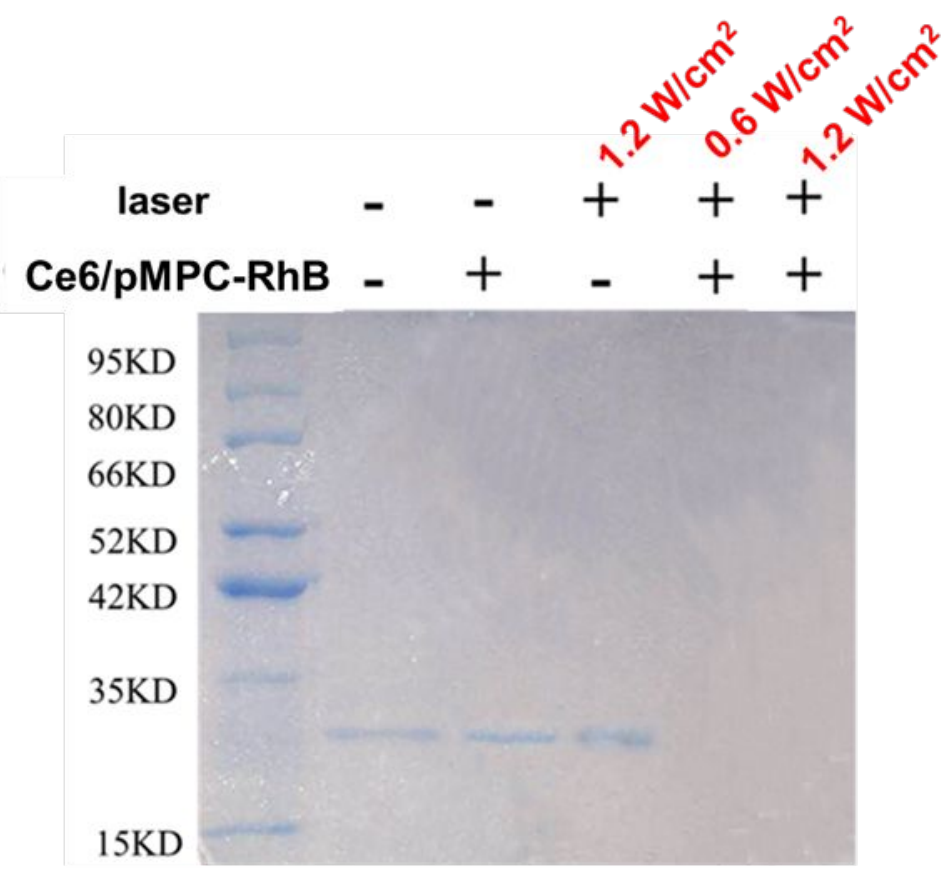

Protein Marker

Supporting information Figure S11. SDS-PAGE analysis of P-gp incubated with different treatments (laser only, Ce6/pMPC-RhB micelles only, and Ce6/pMPC-RhB micelles + different-level laser irradiation). 
On account of the ROS-sensitive property of the P-glycoprotein (P-gp), the production of ROS from Ce6/pMPC-RhB nanomicelles under illumination could break the whole structure of P-gp. Herein, the ROS produced by 1 min irradiation was enough to destroy P-gp structure, thus the last irradiate time in SDS-PAGE analysis was 1 min. As shown in Fig. S11, the non-ROS production group (laser irradiation-only or Ce6/pMPC-DOX micelles-only) exhibited similar P-GP band as the blank control group, while the P-GP band was disappeared in the ROS production group $\left\{\right.$ Ce6/pMPC-RhB nanomicelles plus laser $\left(0.6 \mathrm{~W} / \mathrm{cm}^{2}\right.$ or $\left.1.2 \mathrm{~W} / \mathrm{cm}^{2}\right)$ irradiation $\}$. These result confirmed that even under low-level laser $\left(0.6 \mathrm{~W} / \mathrm{cm}^{2}\right)$ irradiation, the ROS produced by Ce6/pMPC-RhB nanomicelles was capable to destroy the whole structure of P-gp. Moreover, In Western Blotting, the irradiation time was 2 min, which was consistent with the irradiation time of cell treatment. The irradiation time of 2 min was selected in order to ensure that a sufficient amount of ROS was produced to destroy P-gp, while excessive ROS would not be generated.

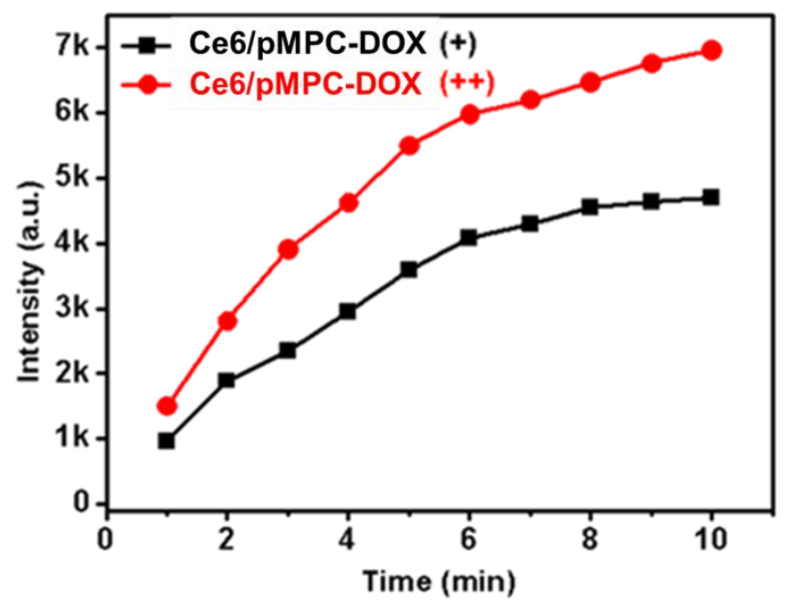


Supporting information Figure S12. ROS generation of Ce6/pMPC-DOX nanomicelles upon laser irradiation with different intensities, where DCFH-DA was applied as ROS detector and PBS was served as blank control (laser irradiation, $+: 0.6 \mathrm{~W} / \mathrm{cm}^{2} ;++: 1.2 \mathrm{~W} / \mathrm{cm}^{2}$ ).

The ROS generation ability of Ce6/pMPC-DOX nanomicelles upon light irradiation was investigated. As shown in Fig. S12, a relatively lower ROS level was observed at $0.6 \mathrm{~W} / \mathrm{cm}^{2}$, suggesting that the relative ROS production be positively correlated with the laser intensity.

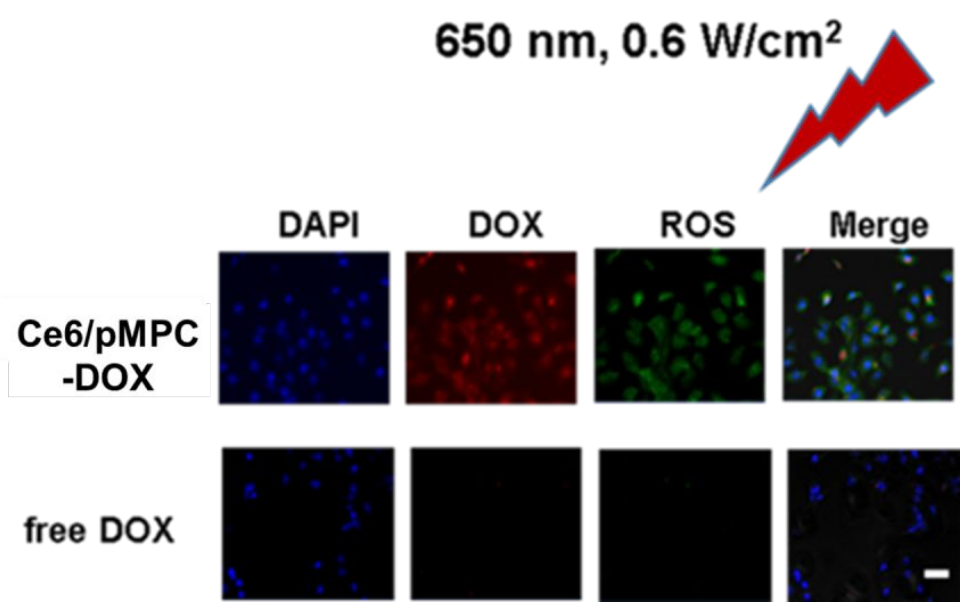

Supporting information Figure S13. Fluorescence images of MCF-7/ADR cells which were incubated with Ce6/pMPC-DOX or free DOX and followed by laser irradiation. Cell nuclei were stained with Hoechest 33258 (blue fluorescence). Red fluorescence indicates DOX accumulation. Green fluorescence indicates ROS generation. Scale bars: $30 \mu \mathrm{m}$.

As shown in Fig. S13, it was found that the red fluorescent DOX merged with the blue fluorescence of cell nuclei, suggesting that the cytoplasm-enriched DOX could efficiently enter the nuclei. 


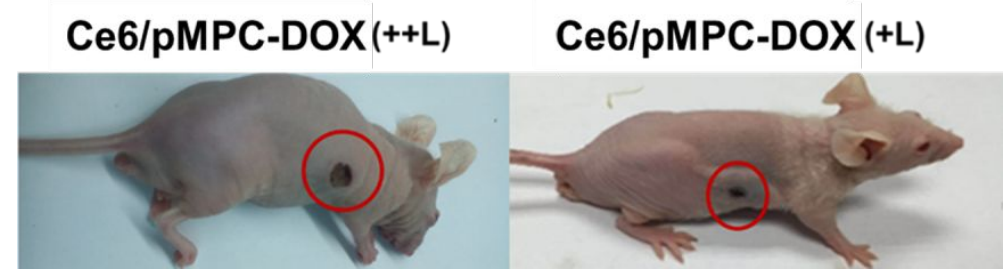

Supporting information Figure S14. Images of MCF-7/ADR tumor-bearing mice which were administrated intravenously Ce6/pMPC-DOX nanomicelles with different intensity laser irradiation (++L: $1.2 \mathrm{~W} / \mathrm{cm}^{2}$; +L: $\left.0.6 \mathrm{~W} / \mathrm{cm}^{2}, 14 \mathrm{~d}\right)$.

As shown in Fig. S14, compared with the mice treated with potency PDT, mice treated with mPDT showed less damage on the skin. Moreover, in vitro experiments, we had confirmed that nanomicelles cannot generate ROS in the absence of laser irradiation (Figure 2A), while the ROS generated under laser irradiation can further destroy P-gp (Figure 3). Therefore, the data difference between the two group of Ce6/pMPC-DOX (without laser) group and Ce6/pMPC-DOX (with laser) group was able to explain that the enhanced effect is from the destruction of P-gp (Figure 5D). In addition, there are some differences between MCF-7 and MCF-7/ADR cells (for example: MCF-7 cells do not express caspase-3 protein while MCF-7/ADR cells are able to express caspase-3 protein ${ }^{1,2}$ ). Therefore, MCF-7 tumor model may not be suitable as a control condition. 

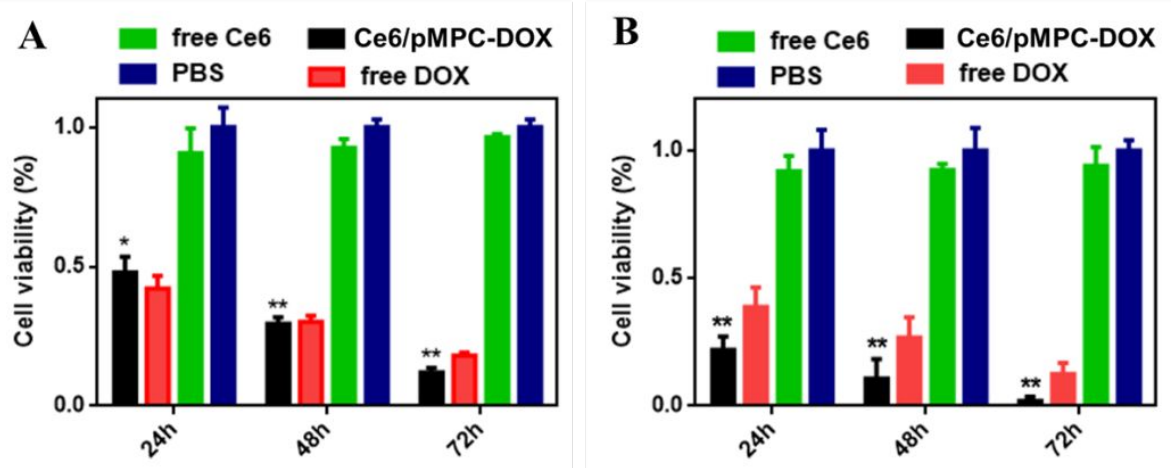

Supporting information Figure S15. Cytotoxicities of different samples at different time points against MCF-7 cells. \{laser: $650 \mathrm{~nm}, 0.6 \mathrm{~W} / \mathrm{cm}^{2}, 5 \mathrm{~min},(\mathrm{~A})$ : without light irradiation, (B): with light irradiation $\}$. Data are presented as the average \pm standard deviation $(n=6)$. The statistical significance level is $* \mathrm{p}<0.05,{ }^{* *} \mathrm{p}<0.01$.

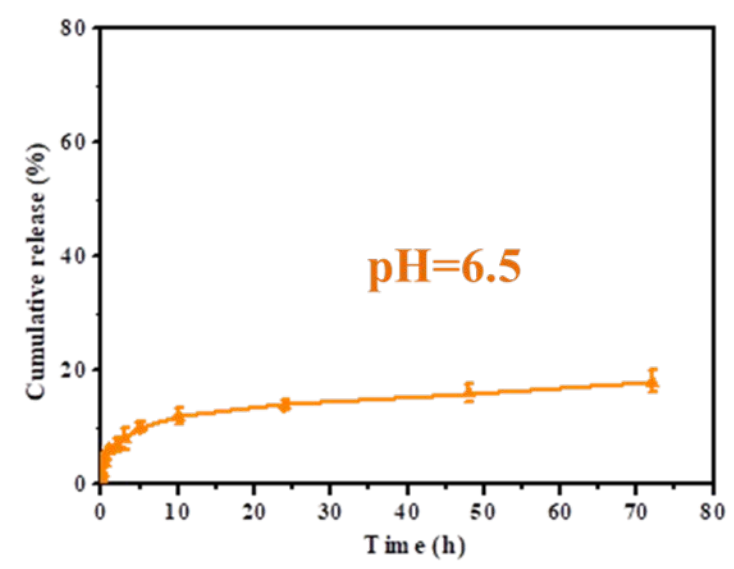

Supporting information Figure S16. DOX release profiles from Ce6/pMPC-DOX nanomicelles in buffer solution $(\mathrm{pH}=6.5)$.

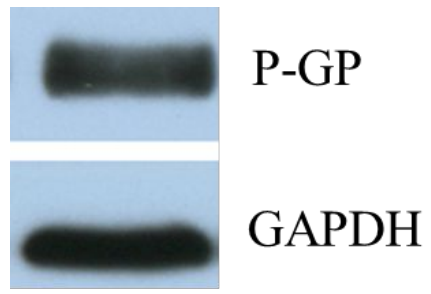

Supporting information Figure S17. Western blot analysis was used to detect the expression of P-gp in tumor tissue cells (MCF-7/ADR tumor-bearing mice). GAPDH was used as a control.

As shown in Figure S17, western blot analysis of proved that we successfully prepared the MCF-7/ADR nude tumor mice. In nude mice experiments, our main purpose was to compare the inhibitory effects of different samples on tumor tissue growth and finally we obtained the corresponding results which were shown in Figure 
5. We did not extend the corresponding experimental time, thus all nude mice were alive at the end of the experiment and the difference was the size of tumor tissue and body weight. In addition, nanomedicine was mainly metabolized by liver and spleen after injection. In our work, the damage of liver and spleen was probable caused by the metabolic process of the broad spectrum anticancer drug DOX.

\section{REFERENCES}

1. Cao, W.; Ma, S. L.; Tang, J. J.; Shi, J. Q.; Lu, Y. J. A Combined Treatment TNF- $\alpha /$ Doxorubicin Alleviates the Resistance of MCF-7/ADR cells to Cytotoxic Treatment. Biochim. Biophys. Acta 2006, 1763, 182-187.

2. Mehta, K.; Devarajan, E.; Chen, J.; Multani, A.; Pathak, S.; Multidrug-Resistant MCF-7 cells: an identity crisis? J. Natl. Cancer Inst. 2002, 94, 1652-1654. 